\title{
Nachbehandlung nach proximalen Femurfrakturen
}

\author{
Nikolaus Schwarz
}

\section{Zusammenfassung}

Die effektive und korrekte physikotherapeutische Nachbehandlung nach orthopädisch-unfallchirurgischen Operationen ist wesentlicher Bestandteil des Behandlungserfolges. Sie fällt deshalb zumindest teilweise in die Kompetenz und Verantwortung des Operateurs. Bei den Patienten handelt es sich in der Mehrheit um Frauen jenseits des 60. Lebensjahres aus städtischen Wohngebieten. Das Behandlungsziel ist die kurzfristige Rückkehr des Patienten in die gewohnte Umgebung und die Wiederaufnahme der Aktivitäten wie vor dem erlittenen Unfall. Die besten Voraussetzungen dafür bieten eine rasche und belastungsstabile chirurgische Versorgung, bei welcher die Frakturteile in weitgehend anatomischer Stellung fixiert werden.
Die am ersten Tag einsetzende Physiotherapie soll die Beweglichkeit der Gelenke erhalten, dem physischen Abbau des Patienten vorbeugen, Behandlungskomplikationen (etwa Pneumonie) verhindern und den Patienten schrittweise zur vollen Mobilität zurückführen. Die Arbeit in geschlossenen Übungsketten bildet dazu eine wesentliche Basis. Der Patient ist gleichzeitig über seine Verletzung und deren Versorgung sowie die daraus folgenden Konsequenzen, etwa die Vorsichtsmaßnahmen nach Implantation einer Hüftendoprothese, zu informieren, um seine Kooperation zu sichern. Geriatrisch-rehabilitative Einrichtungen sind für jene Patienten vorzusehen, denen eine Heimkehr aus dem Akutkrankenhaus nicht zugemutet werden kann.

\section{Einleitung}

Die physiotherapeutische Rehabilitation ist enger Bestandteil der medizinischen Versorgung von Patienten mit Knochenbrüchen. Ganz besonders trifft das auf die meist betagten Patienten $\mathrm{zu}$, welche sich einen Bruch des proximalen Oberschenkelendes zugezogen hatten und bei denen eine Osteosynthese durchzuführen war. Das Behandlungsziel ist, die Patienten soweit als möglich und so rasch als möglich in die Lage zu versetzen, ihr Leben vor dem Unfall wieder aufzunehmen oder an dieses weitgehend anzuschließen.

OP-JOURNAL 2002; 17: 156-159

(c) Georg Thieme Verlag Stuttgart · New York
Der Unfallchirurg stellt die Diagnose nach Verletzung, bestimmt Art und Zeitpunkt der chirurgischen Behandlung, bestimmt Zeitpunkt, Umfang und Intensität des Rehabilitationsprogrammes und den Zeitpunkt des Übertritts von einer Behandlungseinrichtung in eine andere. Wenngleich die Unfallchirurgen selbst die Rehabilitationsbehandlung nicht durchführen und oft auch nicht einmal direkt überwachen, so müssen sie doch die Grundkonzepte verstehen, um mit den Physiotherapeuten kommunizieren zu können und das beste Programm für den individuellen Patienten aussuchen zu können.

Die meisten Patienten mit proximalen Femurfrakturen kommen von zu Hause und haben neben dieser Fraktur keine weitere Verletzung [5]. In unserer Gesellschaft handelt es sich bei den Patienten mehrheitlich um Frauen aus städtischen Wohngebieten. Der soziale Hintergrund ist bezüglich der Wiedereingliederung nach abgeschlossener Behandlung wichtig: $82 \%$ der Patienten nach Schenkelhalsfrakturen in Stockholm kamen direkt aus ihrer Wohnung ins Spital, ebenso viele konnten direkt oder indirekt nach Aufenthalt in einem Institut für Rehabilitation nach erfolgter Behandlung auch wieder nach Hause zurückkehren [5]. Der Rehabilitationserfolg wird durch vorbestehende Krankheiten des Herz-Kreislauf-Systems, des Bewegungsapparates oder des Nervensystems wesentlich negativ mitbeeinflusst.

Die Rehabilitationsprogramme haben die komplette funktionelle Wiederherstellung der verletzten Extremität zum Ziel. Die Voraussetzung dafür ist die Heilung des gebrochenen Knochens in korrekter Position, so dass die Bewegung der Gelenke ohne Einschränkung möglich ist und die Muskeln in vollem Umfang aktiviert werden können. In diesem Sinne bietet die Osteosynthese die grundsätzliche Voraussetzung zur weitestgehenden Rehabilitation durch Einrichtung und Aufrechterhaltung einer korrekten Stellung des gebrochenen Knochens bis zur Bruchheilung bei gleichzeitiger Möglichkeit zur Bewegung der Gelenke und zur Belastung der Extremität. Die Funktion der gebrochenen Extremität sollte in optimaler Weise bereits kurze Zeit nach der Operation wieder uneingeschränkt möglich sein. Das hat zur Voraussetzung, dass die Osteosynthese zu einem frühen Zeitpunkt erfolgt, dass der durch die Operation gesetzte Schaden, insbesondere an den Weichteilen, so gering als möglich ist und dass die Osteosynthese belastungsstabil ist. Viele Patienten mit einer proximalen Femurfraktur sind nicht mehr in der Lage, die Osteosynthese durch Entlastung effektiv zu schützen, so dass eine absolute Belastungsstabilität Ziel der Frakturversorgung sein muss.

Belastungsstabilität ist wesentliche Voraussetzung für eine frühzeitige und optimale Rehabilitation nach proximaler Femurfraktur. 


\section{Belastbarkeit}

Die volle postoperative Belastbarkeit wird durch entsprechende Auswahl der Osteosyntheseverfahren ermöglicht. Unsere Standardverfahren sind in erster Linie intramedulläre Osteosynthesen (Typ PFN). Wenn die mediale Knochenbrücke zwischen beiden Fragmenten intakt ist, also der Trochanter minor nicht ausgebrochen ist, kommt eine Plattenfixation in Betracht (DHS), weil hier ebenfalls Belastungsstabilität zu erzielen ist. Schließlich sind Hüftendoprothesen ebenfalls belastungsstabil und zwar sowohl nach Zementierung als auch solche in pressfit-Technik.

Eine Ausnahme bildet die Osteosynthese der Schenkelhalsfraktur durch Verschraubung in Form einzelner Spongiosaschrauben, Fixation mit diversen Pinmodellen oder mittels der dynamischen Hüftschraube: diese Osteosyntheseformen ergeben nur eine bedingte Belastungsstabilität, welche vom Frakturtyp abhängt: Valgusbrüche, welche verschraubt sind, sind als belastungsstabil zu klassifizieren, Varusbrüche sind nicht belastungsstabil, es ist eine prolongierte Entlastung erforderlich. Daraus resultiert die differenzierte Indikationsstellung in der Form, dass Varusbrüche bei Menschen, die nicht entlasten können, prothetisch versorgt werden und nur bei denjenigen Personen verschraubt werden, denen eine prolongierte Entlastung zugemutet werden kann.

Man muss sich aber beim Problem der Belastbarkeit einer Osteosynthese des proximalen Femurs bewusst sein, dass eine völlige „Entlastung“ nicht möglich ist. Denn schon das Anheben des Beines im Liegen belastet das proximale Femur mit rund 75\% der Vollbelastung beim Gehen [2] und das entlastende Gehen kann nur eine Druckreduktion von ebenfalls $25 \%$ bewirken. Deshalb ist die Anordnung der Entlastung grundsätzlich auf ihre Sinnhaftigkeit zu prüfen.

Eine spezielle Problematik stellen periprothetische Frakturen oder Brüche dar, bei denen aufgrund von Osteoporose und Frakturtyp keine Stabilität erzielt werden kann. In diesen Fällen ist ggf. eine verlängerte Bettruhe unumgänglich, wenn der Patient nicht in der Lage ist zu entlasten.
Mit Ausnahme von Femurhalsverschraubung und Osteosynthese periprothetischer Frakturen sind alle Brüche am proximalen Femur belastungsstabil versorgbar.

\section{Geschlossene/offene Übungskette}

Bewegungen des menschlichen Körpers basieren auf der Aktivierung von Muskeln in unterschiedlicher Reihenfolge und Intensität, wobei $\mathrm{zu}$ jedem Bewegungserfolg ein bestimmter Aktivierungsablauf gehört. Diese koordinierte Aktivierung der Muskeln führt zu einer ebenso koordinierten Bewegung einzelner Gliedabschnitte und wird als kinetische Kette bezeichnet [1]. Es gibt zwei grundsätzlich unterschiedliche Klassen kinetischer Ketten: offen und geschlossen. Bei der offenen Methode ist die Extremität frei beweglich und nicht belastet, bei der geschlossenen Methode werden Hand oder Fuß fixiert oder durch eine äußere Kraft unter Druck gesetzt. Die Bewegung eines Fußes während des Schusses beim Fußballspiel ist ein Beispiel einer offenen Bewegungskette. Muskelaktivierung und Gelenksbewegung sind bei offener bzw. geschlossener Bewegungskette grundsätzlich unterschiedlich.

In der orthopädisch-unfallchirurgischen Rehabilitation wird mit geschlossenen Übungsketten gearbeitet.

In der Rehabilitation von Hüftverletzungen wird mit geschlossenen Bewegungsketten gearbeitet. Es beginnt mit dem Aufsetzen des Fußes auf den Boden, was eine Reihe von Aktivitäten initiiert, um eine optimale Position des Fußes hinsichtlich der Kraftübertragung zu erreichen. Von hier geht die Aktivierung auf die Wadenmuskulatur, welche einerseits den Unterschenkel gegen Ventralverschiebung stabilisiert, andererseits als Teil einer Kraftkette Hüft- und Kniebewegung koordiniert, über. Über eine Stabilisation der Hüfte werden schließlich die Kräfte vom Körper auf den Boden bzw. vom Boden auf den Körper übertragen. Geschlossene Bewegungsketten aktivieren entgegengesetzt wirkende Muskelgruppen, erlauben die Einstellung optimaler Gelenkpositionen, schützen so vor allen Dingen das heilende Gewebe, weil Scher- und Zugkräfte minimiert werden und Fehlbelastungen verhindert werden. Es werden dabei aber nicht alle Muskeln in gleichem Maße eingesetzt und nicht alle Gelenke in vollem Umfang bewegt. So kann etwa die Funktion der
Kniestrecker durch Hüftabduktoren teilweise ersetzt und eine Streckeraktivität vorgetäuscht werden. Es ist Aufgabe der Physiotherapeuten, die Defizite in der funktionellen Rehabilitation der geschlossenen Bewegungskette zu erkennen und durch gezielte Übungen auszugleichen.

Es ist die Aufgabe des Physiotherapeuten zu erkennen, ob alle Muskelgruppen funktionell eingesetzt werden, bzw. muskuläre Defizite auszugleichen.

Die Bewegungen bei der geschlossenen Übungskette sind funktionell und nicht isoliert, wenn auch einzelne Muskelgruppen speziell trainiert werden können. Die Belastung ist physiologisch und betrifft die gesamte kinetische Kette. Die Gelenksbewegung umfasst alle möglichen Bewegungsrichtungen.

\section{Allgemein Aspekte}

Die Rehabilitation proximaler Femurfrakturen hängt von der durchschnittlichen Zeit der Wund- und Frakturheilung ab. Es lassen sich drei Phasen identifizieren:

- Phase 1: 0 bis 4-6 Wochen: Gelenkmobilisation, Muskelaktivierung und Teil- oder Vollbelastung.

- Phase 2: 4 bis 6-12 Wochen: Muskelstärkung und Dauerübungen mit voller Belastbarkeit, Reintegration in das soziale Umfeld.

- Phase 3: 12-24 Wochen: Gangschulung ohne Hilfsmittel, Gehübung auf Boden jeglicher Art, Reintegration in Aktivitäten vor dem Unfall.

Die Rehabilitation der meist betagten Patienten mit isolierten Frakturen des proximalen Femurs erfolgt zwar in einem standardisierten Schema, es müssen aber die funktionellen Möglichkeiten jedes Patienten in Rechnung gezogen werden und das Rehabilitationsprogramm hat sich an den Funktionsstatus, an den Möglichkeiten der Selbstversorgung und der sozialen Aktivität und an den äußeren Lebensumständen vor dem Unfall zu orientieren. Das Ziel ist es, dem Patienten wieder zur Gehfähigkeit und zur Möglichkeit der Selbstversorgung zu verhelfen und den Lebensstil vor dem Unfall wieder aufzunehmen.

Das Outcome ist durch hohes Alter, worunter man ein Alter von über 85 Jahren versteht, negativ beeinflusst. Ebenso negative Faktoren sind der Mangel an sozialem Rückhalt, Aufenthalt in einem Pflegeheim, Gangstörung oder Gangunfähigkeit 
vor dem Unfall, sowie postoperative Komplikationen [4].

Testmethoden bzw. Score-Systeme reduzieren die individuellen Funktionsunterschiede der Patienten auf messbare Parameter. Es können unterschiedliche Aspekte gemessen werden, wie zum Beispiel die Funktion „Sitzen-AufstehenNiedersetzen“. Beim Bartel-Index wird die funktionelle Unabhängigkeit vor und nach dem Unfall gemessen, woraus sich eine Angabe über die Pflegeintensität ergibt. Der Bartel-Index erfasst diejenigen Tätigkeiten, die der Patient im täglichen Leben tatsächlich durchführt und nicht was er alles tun könnte. Es wird die Mobilität in Bezug auf etwaige Einschränkungen in den Alltagsaktivitäten des Patienten, wie Ankleiden oder Toilettebenutzung erfasst. Er dient der Überwachung der Wiederherstellung und dem Vergleich einzelner Patienten an einem Durchschnittskollektiv.

\section{Unmittelbar postoperative Phase}

Das unmittelbare postoperative Rehabilitationsprotokoll hat zum Ziel, Atmungskomplikationen zu vermeiden, den venösen Rückstau zu vermindern, Beinödeme zu reduzieren, Gelenkkontrakturen vorzubeugen und dem allgemeinen physischen Abbau des Patienten vorzubeugen. Der Patient wird unterrichtet über die Lagerung des verletzten Beines und korrekte Bewegungen bei Rückenlage und bei Aufstehen und Niederlegen aus dem Bett. Am ersten postoperativen Tag soll der Patient sich selbständig aufsetzen können und in optimaler Weise auch in der Lage sein, am Bettrand mit $90^{\circ}$ gebeugten Hüft- und Kniegelenken zu sitzen. Der Patient soll über alle Vorsichtsmaßnahmen in Abhängigkeit der durchgeführten Operationstechnik informiert werden. Atem- und Hustübungen werden durchgeführt. Der Patient wird informiert über isometrische Gluteus- und Quadricepsübungen und er soll aktiv die oberen Extremitäten und das nicht verletzte Bein sowie das Sprunggelenk an der operierten Seite bewegen. Es werden die Fußschaufeln beider Beine bei liegendem Patienten kräftig nach oben und unten bewegt, es sollen die Oberschenkel- und Gesäßmuskel für jeweils 10 s angespannt werden, ohne dabei die Beine zu bewegen. In einem nächsten Schritt sollen die Fersen am Bett schleifend gegen den Körper gezogen werden (Abb.1). Wenn der Patient es toleriert, wird mit aktiv assistierten Bewegungsübungen des operierten Hüftgelenkes be-

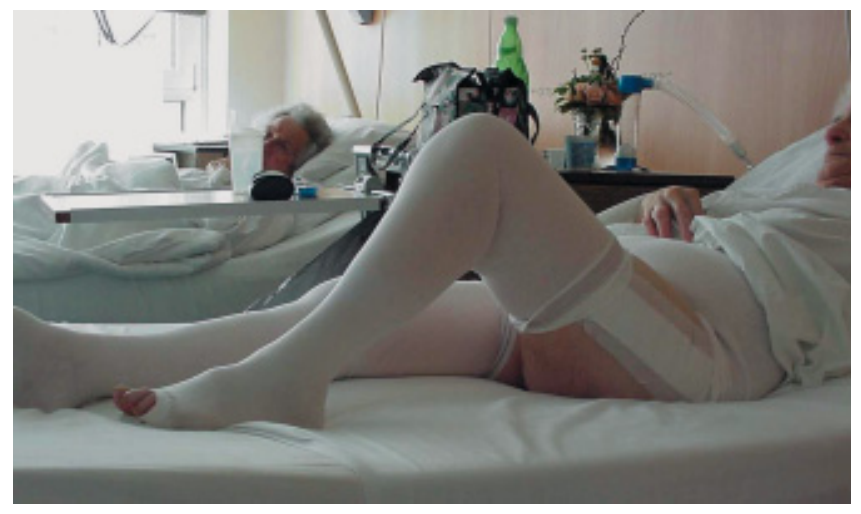

Abb.1 Anziehen des Beines im Liegen mit schleifender Ferse.

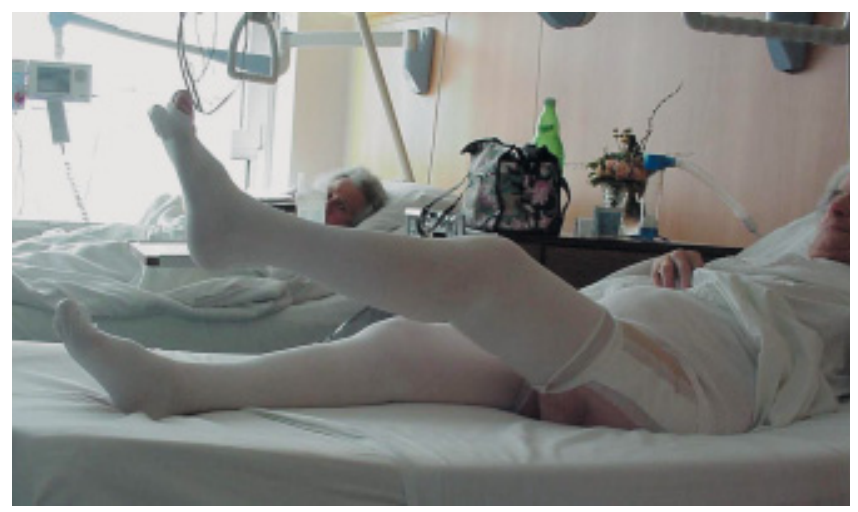

Abb. 2 Freies Beinheben.

gonnen, wobei das Bewegungsausmaß in Beugung und Abduktion schmerzabhängig ist, aber manchmal am ersten Tag schon bis $50^{\circ}$ gehen kann. Am zweiten postoperativen Tag, insbesondere nach der Entfernung der Drainagen, ist eine Stehübung neben dem Bett anzustreben, Beinheben (Abb.2) und Sitzen am Bettrand ist fast immer durchführbar (Abb. 3).

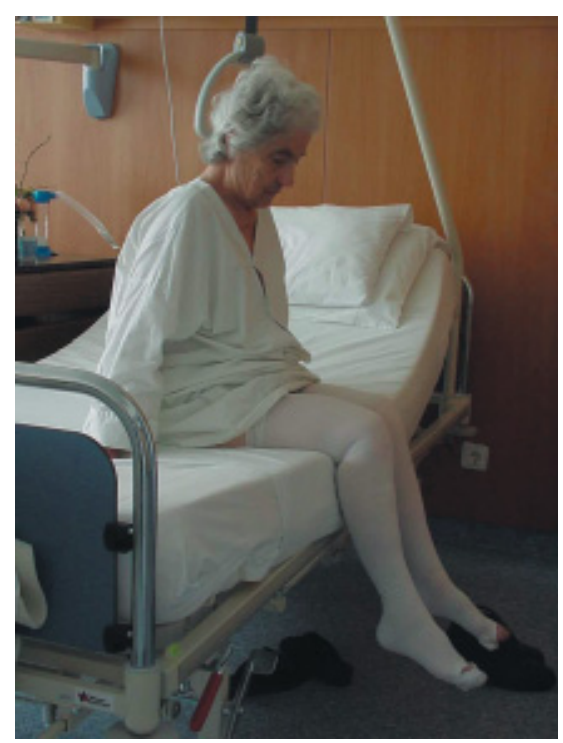

Abb. 3 Sogenanntes Querbettsitzen.
Am dritten postoperativen Tag ist das Ziel, einige Schritte zu machen, eventuell die Toilette zu erreichen und für kurze Zeit in einem Stuhl zu sitzen. Im Sitzen sollen die Kniegelenke wiederholt gestreckt werden. Je nach den individuellen Möglichkeiten ist das Verlassen des Zimmers um den fünften postoperativen Tag und das Stiegensteigen um den zehnten postoperativen Tag das Behandlungsziel.

Die Rehabilitation beginnt am ersten Tag nach der Operation.

Mobilisation nach Hüftendoprothese/ hüftnaher Osteosynthese (nach 3)

- 1. Tag: Atemgymnastik, Armübungen, Fußgymnastik, isometrische Beinübungen, Querbettsitzen,

- 2. Tag: aktive und aktiv assistierte Gymnastik, Steh- und ggf. Gehübungen

5. Tag: Transferübungen, Gangschulung, Bauchlagerung

7. Tag: Stiegensteigen, Glutealtraining 10. Tag: Seitenlage, 4-Punkte-Gang

\section{Gehbehelfe}

Abhängig von der physischen Verfassung des Patienten sind Gehhilfen anzuwenden. Gehbarren (Abb. 4) oder Rollator sind beim älteren Patienten zumindest in den ersten Tagen vorzuziehen, weil sie ein Gefühl erhöhter Sicherheit vermitteln. Vielen Pa- 


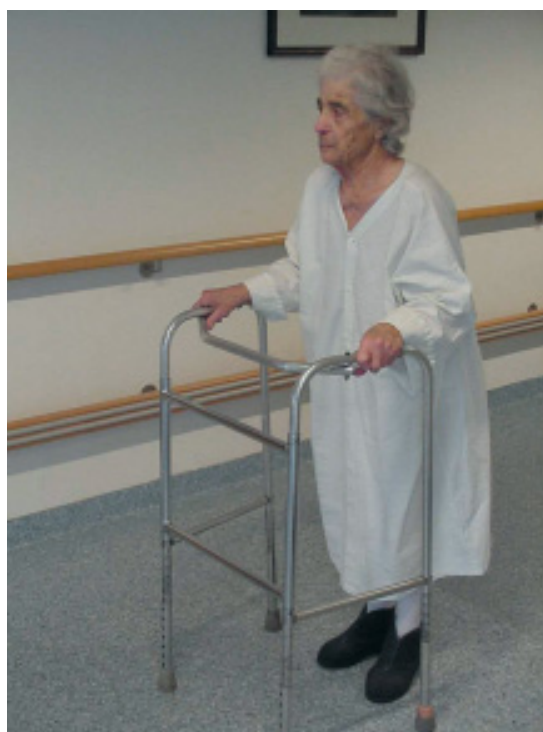

Abb. 4 Gehbarren.

tienten ist es nicht möglich, sich auf Armkrücken zu verlassen (Abb.5), weil sie die dafür nötige Kraft nicht aufbringen. Hier ist ein Versuch, Achselstützkrücken anzubieten empfehlenswert.

Wir setzen Motorschienen (CPM) durchaus auch bei hüftnahen Femurfrakturen zur Mobilisation der Gelenke ein.

\section{Verhalten nach Endoprothesenim- plantation am Hüftgelenk}

Hüftendoprothesen sind zwar belastungsstabil, aber sie sind insbesondere bei Frakturpatienten durch die Gefahr der Prothesenluxation belastet. Eine Reihe von Maßnahmen ist geeignet, die Häufigkeit von Verrenkungen zu vermindern. In den ersten drei Monaten nach der Operation ist die Hüftbeugung über $90^{\circ} \mathrm{zu}$ vermeiden und entsprechend hoch sind alle Sitzflächen einzurichten (Toilette!). Ebenso sind die Überstreckung des Hüftgelenkes und die verstärkte $\mathrm{Ab}$ - und $\mathrm{Ad}$ duktion zu vermeiden. Beim Treppensteigen beginnt man mit dem gesunden Bein und zieht das kranke Bein nach, beim Abwärtsgehen ist es umgekehrt („Gesund geht's bergauf, krank geht's bergab.“). Vorsicht ist beim Gehen auf nassem Boden (Badezimmer) und bei der Körperpflege geboten.

\section{Geriatrisch-rehabilitative Einrichtungen}

Bauliche Voraussetzungen, soziales Umfeld und Versorgungsgegebenheiten im ursprünglichen Lebensbereich der Pa-

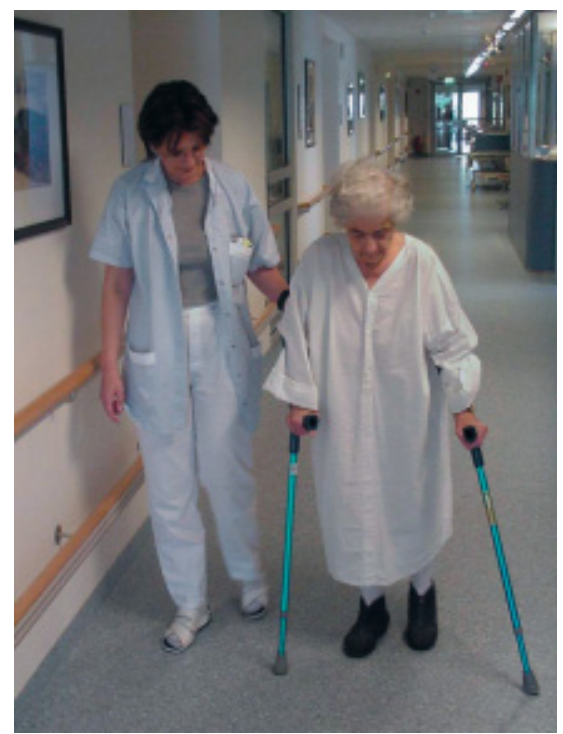

Abb.5 Krückengehen in sichernder Begleitung.

tienten machen eine kurzfristige Rückkehr nach Hause nach einer proximalen Femurfraktur in aller Regel unmöglich. In Österreich gibt es erst seit rund 10 Jahren Ansätze zum Aufbau akutgeriatrischer Einrichtungen, aber man hat erkannt, dass ohne diese die Erfolge in der Rehabilitation deutlich geringer ausfallen. Eine enge Zusammenarbeit mit dem Operateur ist wünschenswert, weil meist nur dieser Komplikationen rechtzeitig erkennen kann und die Indikation zu Revisionsmaßnahmen oder zu Therapieänderungen stellen kann.

Zur Zeit stellt sich die Situation meist so dar, dass die Patienten rasch und umfassend versorgt werden können, dass jedoch geraume Zeit verstreicht, bis postoperativ eine geeignete Rehabilitationsund/oder Pflegestation erreicht wird.

Zum anderen sind die unfallchirurgischen Abteilungen durch einen mehrwöchigen Aufenthalt dieser Patienten meist überfordert und für eine umfassende Rehabilitation nicht ausgestattet.

Die besten Resultate werden jedoch durch spezialisierte Abteilungen mit speziell ausgerichteten Behandlungsregimes erzielt [6]. Diese Autoren konnten durch Umstrukturierung von Abteilung und Therapie die Aufenthaltsdauer reduzieren, die Anzahl der Patienten, die direkt nach Hause zurückkehrten, erhöhen und die Mortalitätsrate senken.

Es liegen hier durchaus Defizite in den Versorgungsmöglichkeiten vor, welche auch angesichts der zunehmenden Alterung der Patienten dringlich behoben werden müssen.

Bei der Behandlung proximaler Femurfrakturen werden die besten Resultate in spezialisierten integrierten chirurgisch-rehabilitativen Abteilungen erzielt.

\section{Schlussfolgerung}

Die Rehabilitation, insbesondere geriatrischer Patienten, nach Frakturen der unteren Extremität bietet für die Klinik eine Reihe von Herausforderungen. Das Verständnis für die altersbezogenen pathophysiologischen Veränderungen und die negativen Effekte verlängerter Bettruhe sind der Ausgangspunkt für die Einrichtung eines adäquaten und sicheren Rehabilitationsprogramms. Funktioneller Status und Lebensumstände vor dem Unfall sind die nicht veränderbaren Voraussetzungen. Das Ziel der Rehabilitation ist die rasche, komplikationsfreie und uneingeschränkte Wiedereingliederung in die physischen und funktionellen sowie sozialen Aktivitäten wie vor dem Unfall.

\section{Literatur}

1 Ben Kibler W, Livingston B. Closed-chain rehabilitation for upper and lower extremities. J Am Acad Orthop Surg 2001; 9: 412-421

2 Bergmann G, Rohlmann A, Graichen F. Invivo-Messung der Belastung von Hüftendoprothesen - Konsequenzen für die Rehabilitation. In E. Hipp, R. Gradinger, R. Ascherl (Hrsg) : Die zementfreie Hüftprothese. Demeter Gräfelfing 1992; 97-103

3 Czurda R. Postoperative Mobilisation an der operierenden Abteilung. Publikation Öster Gesellschaft Rheumatologie 1999; 33-37

4 Flanagan SR, Ragnarsson KT, Ross MK, Wong DK. Rehabilitation of the geriatric orthopedic patient. Clin Orthop 1995; 316: 80-92

5 Holmberg S, Thorngren K-G. Statistical analysis of femoral neck fractures based on 3053 cases. Act.Orthop.Scand. 1987; 218 : $32-41$

6 Parker MJ, Pryor GA, Myles J. 11-year results in 2846 patients of the Peterborough hip fracture project. Act Orthop Scand 2000; 71: $34-38$

Univ.-Prof. Dr. med. Nikolaus Schwarz Facharzt für Unfallchirurgie und Sporttraumatologie Ärztl. Leiter

Unfallkrankenhaus Klagenfurt Waidmannsdorferstr. 35

A-9021 Klagenfurt/Österreich 